\title{
HST/ACS Lyman $\alpha$ imaging of the nearby starburst ESO 338-IG04 ${ }^{\star}$
}

\author{
M. Hayes ${ }^{1}$, G. Östlin ${ }^{1}$, J. M. Mas-Hesse ${ }^{2}$, D. Kunth ${ }^{3}$, C. Leitherer ${ }^{4}$, and A. Petrosian ${ }^{5}$ \\ 1 Stockholm Observatory, AlbaNova University Centre, 10691 Stockholm, Sweden \\ e-mail: matthew@astro.su.se \\ 2 Centro de Astrobiología (CSIC-INTA), E28850 Torrejon de Ardoz, Madrid, Spain \\ 3 Institut d'Astrophysique, Paris (IAP), 98 bis boulevard Arago, 75014 Paris, France \\ 4 Space Telescope Science Institute, 3700 San Martin Drive, Baltimore, MD 21218, USA \\ 5 Byurakan Astrophysical Observatory and Isaac Newton Institute of Chile, Armenian Branch, Byurakan 378433, Armenia
}

Received 14 January 2005 / Accepted 13 March 2005

\begin{abstract}
ESO 338-IG04 (Tololo 1924-416) is a well-known, luminous $\left(M_{V}=-19.3\right)$ Blue Compact Galaxy in the local universe. Its complex morphology indicates a recent merger and/or close interaction, and it contains a central young starburst with compact star clusters of ages $\lesssim 40$ Myr. The galaxy was imaged using the Advanced Camera for Surveys onboard the Hubble Space Telescope (HST) in the Lyman $\alpha$ line and continuum. Using the Starburst99 synthetic spectra and other imaging data from the Wide Field and Planetary Camera 2, we developed a technique that allows us to make the first photometrically valid subtraction of continuum from the Ly $\alpha$ line. The method allows us to disentangle the degenerate effects of age and reddening by careful sampling of the UV continuum slope and $4000 \AA$ discontinuity. Our results are in qualitative agreement with the models of $\operatorname{Ly} \alpha$ escape being regulated by kinematical properties of the interstellar medium. The line-only image shows Ly $\alpha$ in both emission and absorption. Most notably, Ly $\alpha$ emission is seen from central bright young clusters and is in spatial agreement with features present in a longslit spectrum taken with the Space Telescope Imaging Spectrograph. Ly $\alpha$ is also seen in diffuse emission regions surrounding the central starburst where photons escape after one or more resonant scatterings in HI. Quantitative photometry reveals a total flux in the Ly $\alpha$ line of $f_{\mathrm{Ly} \alpha}$,TOT $=194 \times 10^{-14} \mathrm{erg} \mathrm{s}^{-1} \mathrm{~cm}^{-2}$. The Ly $\alpha$ flux in a $10 \times 20^{\prime \prime}$ elliptical aperture centred on the brightest central star cluster measures $f_{\mathrm{Ly} \alpha, \mathrm{IUE}}=134 \times 10^{-14} \mathrm{erg} \mathrm{s}^{-1} \mathrm{~cm}^{-2}$ with an equivalent width of $22.6 \AA$. This is in close agreement with previous studies made using spectra from the IUE satellite to which our aperture was created to match. Thus we demonstrate that we have software in place to create line-only Ly $\alpha$ maps of nearby galaxies. Analysis of parameter dependencies show our technique to be largely parameter independent, producing Ly $\alpha$ maps indistinguishable from one another by eye and with Ly $\alpha$ fluxes consistent with one another to better than $50 \%$. We see large amounts of diffuse $\operatorname{Ly} \alpha$ emission that dominates the total $\operatorname{Ly} \alpha$ output which are interpreted as centrally produced Ly $\alpha$ photons scattered by neutral hydrogen. By comparison of $\mathrm{Ly} \alpha$ fluxes with $\mathrm{H} \alpha$ fluxes of a previous study, we estimate that each observed Ly $\alpha$ photon has undergone $\gtrsim 2$ additional scatterings. We see that Ly $\alpha$ line kinematics closely correlate with other kinematic tracers but, within these data, find no evidence for Ly $\alpha$ emission or absorption from star clusters being a function of age.
\end{abstract}

Key words. galaxies: starburst - galaxies: individual: ESO 338-IG04 - galaxies: ISM - ultraviolet: galaxies - X-rays: stars

\section{Introduction}

The potential importance of the Ly $\alpha$ emission line for probing galaxy formation and evolution in the early universe has long since been recognised. Partridge \& Peebles (1967) suggested the $\operatorname{Ly} \alpha$ line to be an important spectral signature in young galaxies at high redshift since the expected Ly $\alpha$ luminosity should amount to a few percent of the total galaxy luminosity. If so, Ly $\alpha$ fluxes of order $10^{-15} \mathrm{erg} \mathrm{s}^{-1} \mathrm{~cm}^{-2}$ should be detectable in galaxies forming stars with rates of $10^{2} M_{\odot} \mathrm{yr}^{-1}$ at redshifts $(z)$ around 3. Early observational attempts were largely

* Based uon observations with the NASA/ESA Hubble Space Telescope obtained at the Space Telescope Science Institute, which is operated by the Association of Universities for Research in Astronomy, Inc., under NASA contract NAS5-26555. disappointing and demonstrated the scarcity of luminous $\operatorname{Ly} \alpha$ emitters at high redshifts (Pritchet 1994); however, the situation has changed dramatically in recent years. Candidate Ly $\alpha$ emitters are now routinely detected at redshifts $z>5$ using narrow-band imaging techniques, although the actual numbers of detected Ly $\alpha$ emitters at high redshift are smaller than was initially predicted (Frye et al. 2002; Fujita et al. 2003; Ouchi et al. 2003; Malhotra \& Rhoads 2002; Fynbo et al. 2001).

In theory, Ly $\alpha$ can be used to quantitatively examine reionisation, star-formation rates, and correlation functions, along with grouping parameters in the distant universe. However, in order to proceed, it is essential for us to understand the physics that regulates $\operatorname{Ly} \alpha$ escape. The situation is complicated by a number of factors, not the least that Ly $\alpha$ lies in the far ultraviolet (UV) domain, making it impossible to observe from the 
ground at redshifts less than $\sim 2$. Furthermore, Ly $\alpha$ is a resonant line and therefore sensitive to multiple scatterings and attenuation in HI clouds and galactic halos. This is evident from the Ly $\alpha$ forest and damped Ly $\alpha$ absorption seen in QSO absorption spectra. Multiple scatterings can attenuate Ly $\alpha$ via two mechanisms. Firstly, resonant scatterings redirect the Ly $\alpha$ photons, thereby increasing the path length on which the Ly $\alpha$ photons escape the neutral cloud with respect to the nearby continuum. This serves to increase the probability that the photons will be destroyed by interactions with dust grains. Secondly, scattering of Ly $\alpha$ photons by HI can result not in one Ly $\alpha$ photon but two photons of lower energy. The probability of this two-photon emission is mildly temperature-sensitive but always $\sim 1 / 3$.

Early ultraviolet observations of a few nearby starbursts appeared to confirm the high- $z$ result: Ly $\alpha$ was surprisingly weak; weaker still in more dust-rich galaxies. The reason was first thought to be multiple scatterings in HI gas increasing the probability of dust absorption (Neufeld 1991); however, subsequent Hubble Space Telescope (HST) observations showed that the problem is much more complex. Kunth et al. (1998) discussed a set of eight nearby star-forming galaxies observed with the Goddard High Resolution Spectrograph (GHRS) on HST. Four galaxies showed clear Ly $\alpha$ P-Cygni profiles indicating largescale outflows of the interstellar medium (ISM). In contrast, the four other galaxies show damped Ly $\alpha$ absorption, regardless of their dust content. Thuan \& Izotov (1997) studied the unique starburst Tol1214-277 whose very strong and symmetric Ly $\alpha$ line shows no trace of blueshifted absorption. A subset of the analyzed starburst galaxies were observed with STIS (Mas-Hesse et al. 2003), in order to understand the kinematical structure of the outflowing gas. The main observational result of this work was the identification of rather large expanding shells of neutral gas, spanning up to several kiloparsecs and covering a much larger area than the starburst itself.

The complex nature of Ly $\alpha$ escape revealed by the GHRS and STIS spectroscopy (Kunth et al. 1998; Mas-Hesse et al. 2003) raised additional issues. In the case where the UV source is shielded by a slab of static neutral hydrogen, the galaxy appears as a damped Ly $\alpha$ absorber. However, if the dust content is small the Ly $\alpha$ photons may, after multiple resonant scatterings, diffuse out over a larger area, although attenuated by the twophoton process. This would create a bias in spectroscopic studies which usually target the regions of peak UV intensity, i.e. in this scenario, the places from which we do not expect to see Ly $\alpha$ in emission. Another possibility is that the UV-continuum sources are partly shielded by a clumpy medium, in which case we would see a mixture of absorption and emission. In cases where the ISM has a non-zero radial velocity with respect to the UV continuum source, Ly $\alpha$ will appear in emission with a characteristic P-Cygni profile. Since radial velocity of the ISM is so important in determining $\operatorname{Ly} \alpha$ escape, morphology and kinematics of the galaxy play a vital role as certain kinematic configurations may enhance the escape probability. This in turn implies that the Ly $\alpha$ escape probability can vary greatly across the starburst region and, for example, during a merger the escape probability may be enhanced. ESO 338-IG04 is a perfect galaxy to study in this respect because it exhibits Ly $\alpha$ that has escaped via all of the mechanisms listed.
In the distant universe, Ly $\alpha$ imaging and low resolution spectroscopic techniques are now successfully used to find large numbers of Ly $\alpha$ emitting galaxies. However, without proper understanding of the $\operatorname{Ly} \alpha$ emission processes this line cannot be used to estimate star formation rates and Ly $\alpha$ becomes a dubious choice to use when studying clustering if the biases are not properly known. If the star-forming activity of a high redshift galaxy is connected with its environment, e.g. induced by merger or interaction, the Ly $\alpha$ escape probability will not be independent of this parameter.

These considerations led us to start a pilot program to image local starburst galaxies in the Ly $\alpha$ line using the solar blind channel (SBC) of the Advanced Camera for Surveys (ACS) on HST; thereby allowing us to study the Ly $\alpha$ emission and absorption morphology in detail. A sample of six galaxies with a range of luminosities $\left(M_{V}=-15\right.$ to -21$)$ and metallicities $\left(0.04 Z_{\odot}\right.$ to $\left.\sim Z_{\odot}\right)$ were selected, including previously known Ly $\alpha$ emitters, as well as absorbers. A lower velocity limit of $c z \geq 2500 \mathrm{~km} \mathrm{~s}^{-1}$ was required so that the Ly $\alpha$ line could easily be differentiated from the bright geocoronal Ly $\alpha$ line in any spectroscopic observations. The sample was observed during 30 orbits in Cycle 11. The observations were obtained with the F122M filter for Ly $\alpha$ online imaging and F140LP as a continuum filter.

The first results for two of the observed galaxies, ESO 350-IG38 and SBS 0335-052, were presented in Kunth et al. (2003). In that study, we subtracted the continuum by using the relative instrument sensitivities (PHOTFLAM values) of the two instrument configurations, using the continuum slope $(\beta)$ obtained from IUE or GHRS data for the bright knots and assuming a flat continuum in all other regions. However, these assumptions are not sufficient to perform an accurate photometric analysis and allowed the authors to obtain only a qualitative picture of the $\operatorname{Ly} \alpha$ morphology.

In this paper we focus on the most nearby galaxy in the sample, the well-known luminous blue compact galaxy ESO 338-IG04. For this galaxy, Wide Field and Planetary Camera 2 (WFPC2) images in numerous optical passbands and Space Telescope Imaging Spectrograph (STIS) spectroscopy are also available. Using these we developed methods for accurate continuum subtraction, which allowed quantitative analysis of the Ly $\alpha$ emission and absorption.

\subsection{Short facts about the target: ESO338-IGO4}

ESO 338-IG04 (Tololo 1924-416) is a well-known luminous $\left(M_{V}=-19.3\right)$ blue compact galaxy (BCG) in the local universe $\left(v_{\mathrm{rad}}=2830 \mathrm{~km} \mathrm{~s}^{-1}\right.$, Cannon et al. 2004) and can be seen in Fig. 1. The galaxy was first studied in detail by Bergvall (1985). The nebular oxygen abundance is $12+\log (\mathrm{O} / \mathrm{H}) \approx 8.0$ (Östlin et al. 2003).

Images obtained with HST's Faint Object Camera (FOC) show that the centre of ESO 338-IG04 hosts a population of UV-bright star clusters, just like many other starbursts (Meurer et al. 1995). Subsequent WFPC2 observations have increased the number of known clusters to more than 100 and have shown them to span ages from just a few Myr to several Gyr 


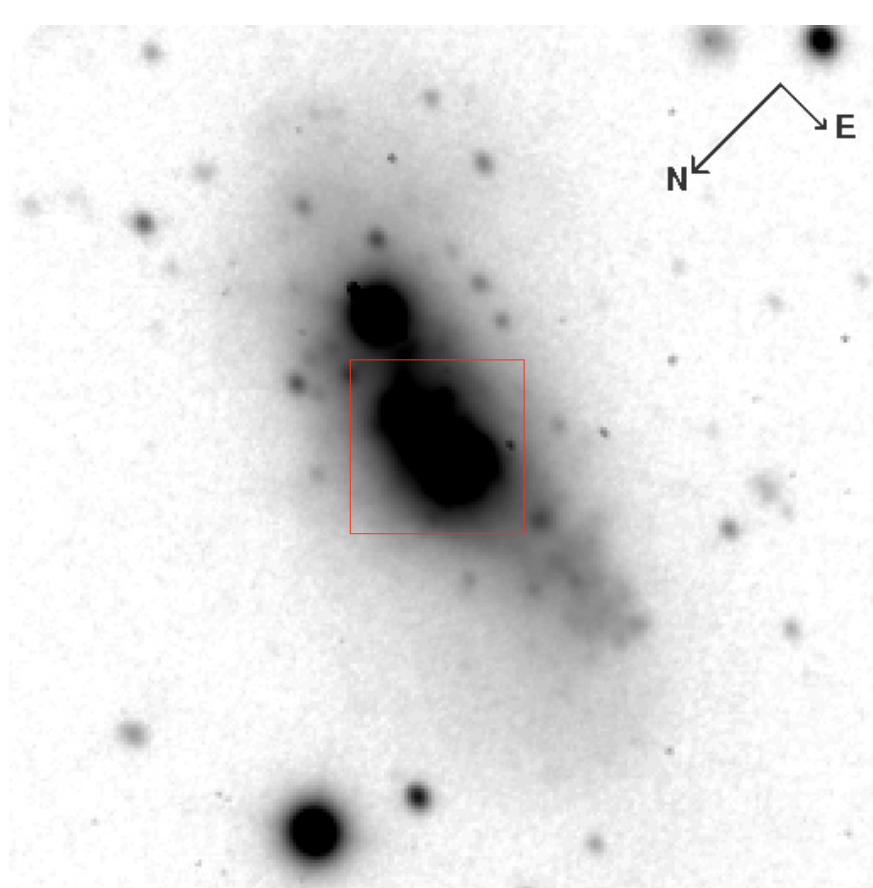

Fig. 1. Ground-based $R$-band image of ESO 338-IG04 taken with the EMMI instrument of the NTT on La Silla (Bergvall \& Östlin 2002). The image is $70 \times 70^{\prime \prime}$ with the longer north arrow of the compass representing $10^{\prime \prime}$. The image has been rotated to the same orientation as the ACS images. The box in the centre of the galaxy shows the $15 \times 15^{\prime \prime}$ region shown in Fig. 2 .

(Östlin et al. 1998, 2003). The age distribution of the young clusters indicates that the present starburst was initiated 40 Myr ago. Spectroscopic observations from the International Ultraviolet Explorer (IUE) showed ESO 338-IG04 to be a bright Ly $\alpha$ emitter with a UV continuum that has close to zero internal dust extinction (Giavalisco et al. 1996; Calzetti et al. 1994).

ESO 338-IG04 has a perturbed $\mathrm{H} \alpha$ velocity field (Östlin et al. 1999, 2001) and morphology (Bergvall \& Östlin 2002) suggesting that the galaxy has been involved in a merger/strong interaction with a small galaxy. The presence of a companion galaxy (Östlin et al. 1999) and extended HI emission between the two (Cannon et al. 2004) further supports this starburst triggering scenario. The galaxy is a true starburst in the sense that the time-scales for gas consumption and build-up of the observed stellar mass with the current SFR are both $\sim 1$ Gyr, i.e. much shorter than the age of the universe (Östlin et al. 2001).

The bright Ly $\alpha$ line, the existence of HST data at other wavelengths, and the small internal reddening made ESO 338IG04 a perfect target for a Ly $\alpha$ imaging study with ACS.

\section{Data acquisition and basic reductions}

\subsection{ACS observations}

Our observations were obtained with the Solar Blind Channel of the ACS under general observer (GO) program 9470 with two filters: $F 122 M$ (Ly $\alpha$ on-line) and F140LP (as a continuum filter). The ACS/SBC was the ideal instrument mode for these observations because its field of view is appropriate for encompassing the galaxies comfortably; and it utilises a MAMA detector and is thus not susceptible to cosmic rays. The target galaxy was observed for 5 orbits, with the F122M images obtained during the SHADOW part of the orbit in order to lower the geocoronal background. The remainder of the orbit was then dedicated to obtaining the F140LP images. The geocoronal background is low in both filters but is almost always brighter than the signal in the online image from the target galaxies. The total integration times per galaxy in the F122M and F140LP filters were 9095 and 3000 s, respectively. Observations in each filter were split into five separate exposures. The drizzled images produced by the standard pipeline (with rectified pixel scale of $0.025^{\prime \prime} \mathrm{pix}^{-1}$ ) were combined after coalignment; and the background in each filter was subtracted using a first degree polynomial surface. These images were then corrected for Galactic interstellar extinction with the Cardelli law (Cardelli et al. 1989). Values used were $E(B-V)=0.087$ and $A_{B}=0.375$ (Schlegel et al. 1998). The solid angle subtended by ESO 338-IG04 is so small that these values can be assumed over the entirety of the images.

\subsection{Supporting HST observations}

In addition to the ACS images we make use of images in the $F 218 W, F 336 W, F 439 W, F 555 W$, and $F 814 W$ filters obtained with the Planetary Camera (PC) aperture of the WFPC2 under GO program 6708. These observations have been used to study bright compact star clusters and are described in Östlin et al. (1998, 2003). We use these images aligned and rebinned to the same orientation and scale as the ACS data. They were also corrected for Galactic interstellar extinction at the central wavelengths of the filters. A narrowband image of the $[\mathrm{OIII}]_{\lambda 4959} \AA$ emission line has been obtained with HST/STIS in GO program 8668 (Östlin et al. in prep.). This observation takes advantage of the fortuitous redshift of ESO 338-IG04 where the $4959 \AA$ line is redshifted to $5007 \AA$ and can be imaged with the very narrow $(F W H M=5 \AA)$ STIS/F28X50OIII filter centred on $5007 \AA$. We also make use of a HST long-slit spectrum obtained with the Space Telescope Imaging Spectrograph (STIS) with the G140L grating and $0.2^{\prime \prime}$ wide slit, under GO program 9036. The slit was placed approximately along the major axis of the target as shown in Fig. 2.

\section{Subtracting the continuum}

We use the F140LP filter to subtract the continuum from $F 122 M$ and produce a line-only Ly $\alpha$ image. As with any continuum subtraction, the continuum image is used to estimate the flux due to continuum processes at the wavelength of the line. It is necessary to scale the continuum image to compute the continuum level at the the line. We define the Continuum Throughput Normalisation (CTN) factor as the ratio of flux in the $F 140 L P$ to that in the F122M filter for a given spectral energy distribution (SED), hence with no Ly $\alpha$ feature in emission or absorption:

$\left(f_{F 122 M} \times \mathrm{CTN}\right)-f_{F 140 L P}=0$

where $f_{F 122 M}$ and $f_{F 140 L P}$ are the $f_{\lambda}$ fluxes in the $F 122 M$ and F140LP filters, respectively. 


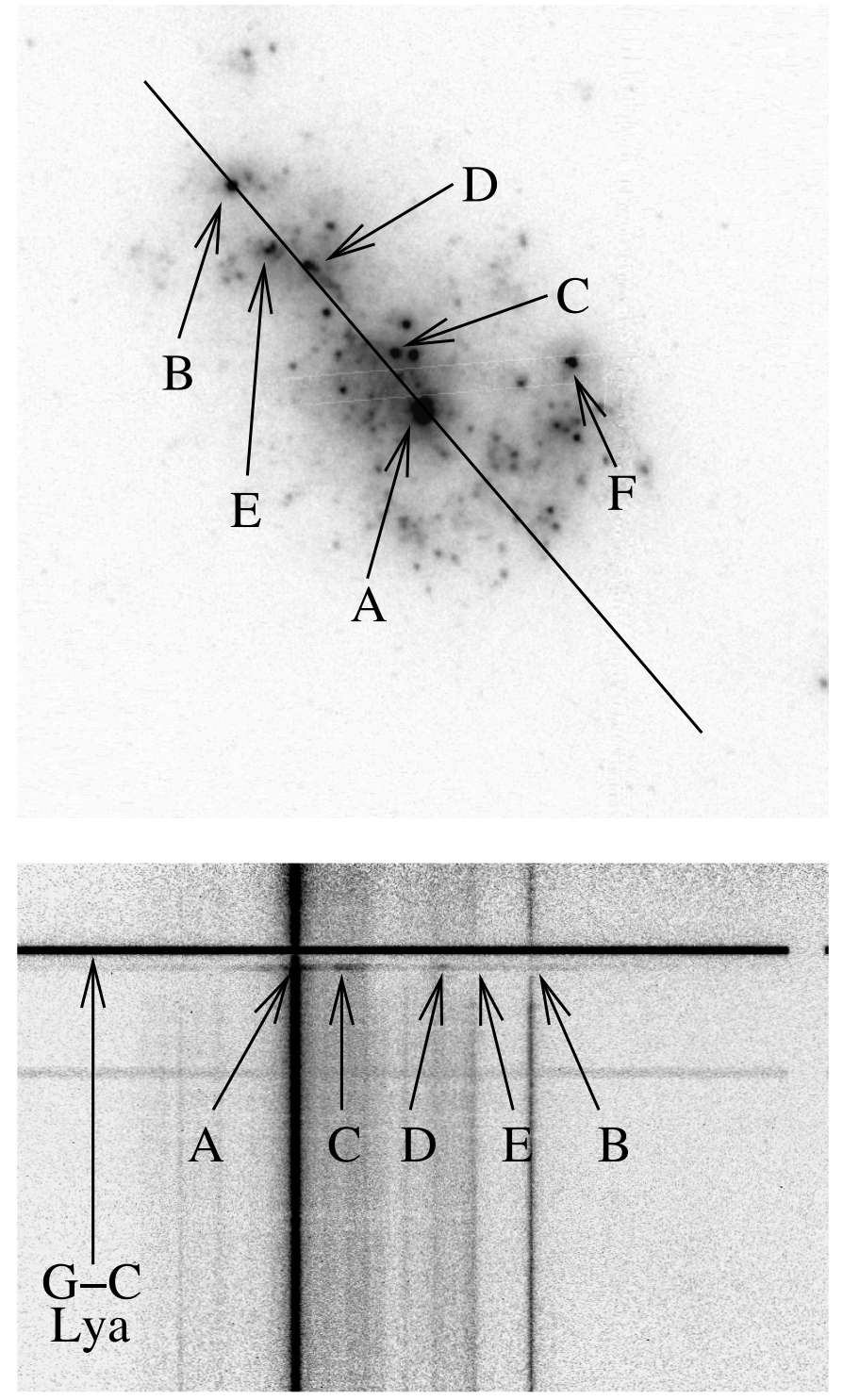

Fig. 2. Top: ACS/F140LP image with the STIS slit position overlayed and certain features labeled. The image size is $15 \times 15^{\prime \prime}$. This image corresponds in size and orientation to the box in Fig. 1 and shows how the bright UV continuum radiation emanates from a much smaller region than the observed size of ESO 338-IG04 in optical bandpasses. The nearly horizontal lines running across the centre of the image are due to the mosaic of the detector and couldn't quite be removed with our dithering pattern. Bottom: STIS G140L spectral image for the slit position indicated above. $\lambda$ decreases with increasing ordinate. Two geocoronal lines are visible running horizontally: the strong Ly $\alpha$ line can be seen towards the top of the image and the fainter OI line at $1304 \AA$ further down. The labels denote the same spatial regions as in the upper panel and point at the $\operatorname{Ly} \alpha$ wavelength in the restframe of the target.

If one knows the instrument sensitivities in these filters and the SED between these wavelengths, the CTN factor can be readily computed. The simplest method to obtain a reasonable first order estimate of CTN factor is to assume the SED to be a power-law governed by index $\beta$ such that $f_{\lambda}=\lambda^{\beta}$. In Kunth et al. (2003) we used such an assumption to make $\operatorname{Ly} \alpha$ continuum subtractions for the first two galaxies in the

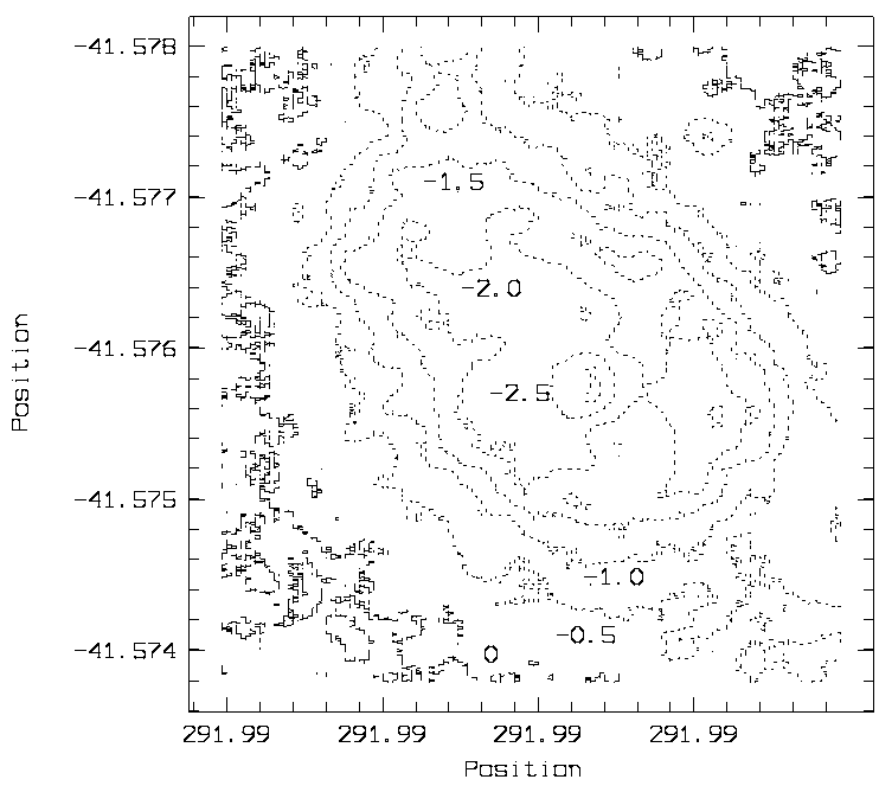

Fig. 3. Contour plot showing the spatial variation of the UV continuum slope $\beta$ between the ACS/F140LP filter at $1527 \AA$ and the WFPC2/F218W filter at $2204 \AA$. The innermost contour corresponds to the steepest slope of $\beta=-3$. Subsequent contours are spaced at equal intervals of 0.5 . The image covers $15 \times 15^{\prime \prime}$. The image has been smoothed using a $10 \times 10$ pixel boxcar filter.

sample, ESO 350-IG38 and SBS 0335-053. There we used available IUE spectra and WFPC2 $V$-band images to estimate global values of $\beta$ and used these values to subtract the continuum in those regions dominated by bright clusters. Assuming standard calibration and a flat continuum $(\beta=0)$, the CTN is simply the ratio of the PHOTFLAM keywords of the offand online instrument configurations: 10.33 . This value was then used to subtract the continuum in the remaining (non starcluster) regions. As a first approximation, this method provided a reasonable map of $\operatorname{Ly} \alpha$ emission and absorption and enabled the authors to discuss the kinematics of the line. It is, however, highly dependent upon the validity of the assumptions and doesn't allow any accurate photometric study to be performed. Furthermore this method doesn't properly allow for spatial variation in the slope of the continuum - a quantity that is highly sensitive to local variations in burst age and internal dust reddening.

For ESO 338-IG04, the UV continuum slope was mapped by using the ACS/F140LP image and a variety of WFPC2 images at longer wavelengths. A resulting $\beta$-map, constructed using the $F 218 \mathrm{~W}$ filter, can be seen in Fig. 3. As the map shows, $\beta$ reaches a minimum of -3 in the central bright star cluster, indicating a steep continuum dominated by $\mathrm{O}$ and $\mathrm{B}$ stars. $\beta$ increases to values of $-0.5 \lesssim \beta \lesssim 0.5$ away from the starburst region, i.e. an approximately flat continuum.

Using such a map of the continuum slope to subtract the continuum assumes that the UV continuum between $1527 \AA$ and $\operatorname{Ly} \alpha$ remains an unbroken power law. Preliminary continuum subtractions with this technique showed strong Ly $\alpha$ absorption throughout the regions of bright continuum. This is contradictory to the STIS spectra extracted from these 
regions, which show Ly $\alpha$ in emission and is indicative of the fact that this technique causes the subtraction of too much continuum. This discrepancy can be attributed to a number of effects: firstly, examination of the IUE spectrum suggests that the continuum slope deviates from the power-law, flattening out at wavelengths shortward of $\sim 1400 \AA$. Secondly, the presence of discrete stellar Ly $\alpha$ absorption features may also serve to reduce the total Ly $\alpha$ output from a region. Often most importantly, inspection of the STIS spectra shows that Galactic absorption of $\operatorname{Ly} \alpha$ is significant in the regions where the continuum is strong, causing a significant reduction of the flux in the online filter. While the assumption of a power-law continuum may be sound redwards of $\operatorname{Ly} \alpha$, it fails to address any effects that may arise as a result of Galactic HI and some technique that accounts for this is essential.

\subsection{Synthetic spectra}

In order to tackle some of the issues raised above, we began to explore the properties of the Starburst99 synthetic spectral models (Leitherer et al, 1999). These models were selected because they use the most appropriate UV stellar atmospheres and cover a large parameter space enabling us to test how different model parameters may affect our final results. The possibility also exists to run one's own simulations, based upon exact requirements, and to interface with the Mappings code (Kewley et al. in prep.) for the inclusion of nebular emission lines. The initial model set selected included stellar and nebular emission for an instantaneous burst of metallicity $Z=0.001$ and Salpeter IMF. The metallicity of ESO 338-IG04 is known to be around $10 \%$ solar (Bergvall 1985; Masegosa et al. 1994) so the lowest available metallicity was chosen. Östlin et al. (2003) showed that a Salpeter IMF extending up to $\sim 100 M_{\odot}$ was the best fit to their data on this galaxy. The effects of varying these parameters are addressed in Sect. 5. The models were used to investigate the CTN factor and optical colours with the effects of aging and dust reddening.

One feature that should be the same in all spectra is the Galactic Ly $\alpha$ absorption which removes a fixed fraction of photons near $1216 \AA$ A. Since ESO 338-IG04 covers a small solid angle on the sky (less than half a square arcmin), we assume that the column density of Galactic HI along the sightline is constant over the face of the galaxy. A 1D STIS spectrum of the central star cluster (where continuum and signal-to-noise are highest) was extracted. Using the XvoigT program (Mar $\&$ Bailey 1995) we made a single-component fit of a Voigt profile to the wings of the Galactic absorption profile. This yielded a Galactic HI column density of $\log (N(\mathrm{HI}))=20.7 \mathrm{~cm}^{-2}$, in close agreement with the value of $\log (N(\mathrm{HI}))=20.8 \mathrm{~cm}^{-2}$ from Galactic HI maps of Dickey \& Lockman (1990). This normalised Voigt profile was then used to apply the Galactic Ly $\alpha$ absorption profile to the synthetic spectra by simple convolution. A fit of the Voigt profile to the wings of the absorption profile can be seen in the upper panel of Fig. 4.

Milky Way interstellar reddening also has the same affect on any spectrum since reddening simply removes a fraction of the flux at a given wavelength. The Galactic extinction law of Cardelli et al. (1989) was used with the parameters
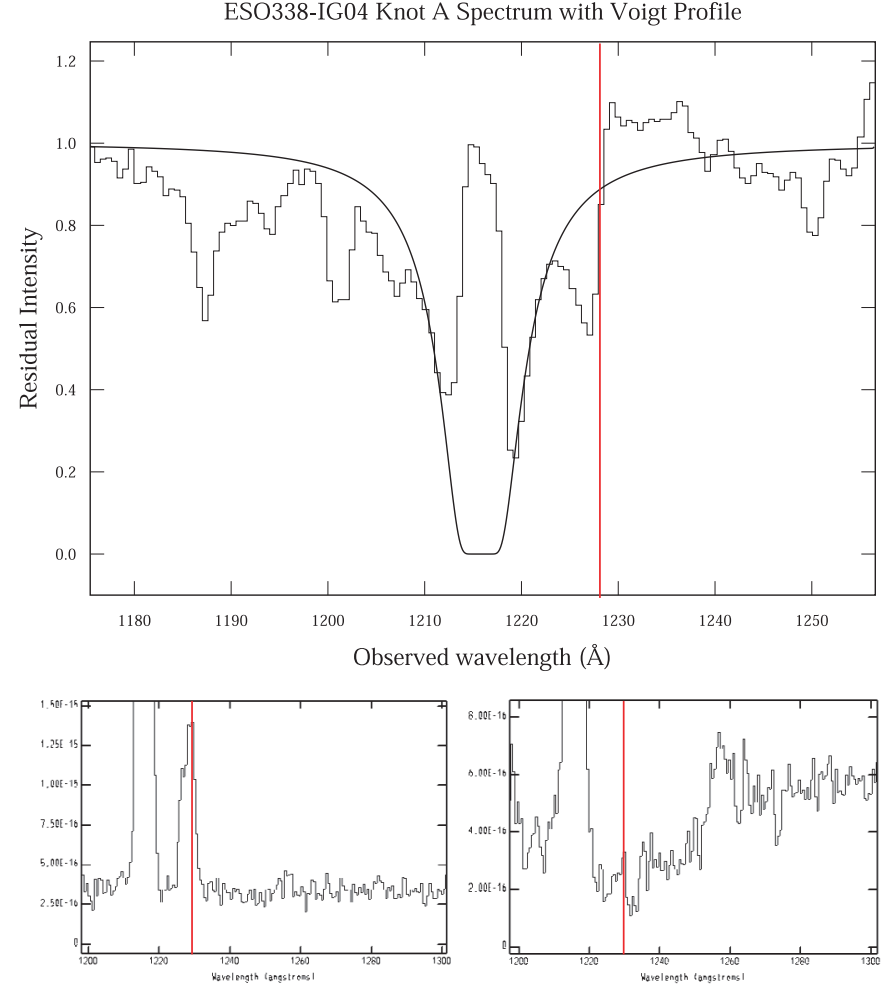

Fig. 4. Top: part of the STIS G140L spectrum for knot A with a Voigt profile fitted to the wings of the damped Galactic Ly $\alpha$ absorption profile, giving a Galactic HI column density of $20.7 \mathrm{~cm}^{-2}$. The strong geocoronal Ly $\alpha$ line can clearly be seen at $1216 \AA$. The vertical line at $1228 \AA$ shows the wavelength of Ly $\alpha$ in the target galaxy and blueshifted absorption can clearly be seen just shortwards of this wavelength. Flux units are normalised to the continuum at $1300 \AA$. Bottom: STIS spectra covering 1200 to $1300 \AA$ from knots C (left) and B (right) where Ly $\alpha$ is seen in emission and absorption respectively. The absorption trough extends from around the geocoronal line to wavelengths as long as $1250 \AA$.

of $A_{\mathrm{V}}=0.288$ and $E(B-V)=0.087$ (taken from the values quoted in NED, calculated from Schlegel et al. 1998) to redden all the spectra.

Our investigation was mainly concerned with how age and internal reddening affect the CTN factor and broadband UV/optical colours when computed from synthetic spectra. We obtained the instrument throughput sensitivity profiles for all of our instrument configurations from the SYNPHOT package in the IRAF/STSDAS. This allowed us to compute synthetic fluxes from the spectra in all of our passbands by simple integration of the spectrum convolved with the instrument sensitivity profile. Conversion to the Vega magnitude system was performed using the quoted zeropoint offsets. Since emission lines are not included in the spectra (no $\operatorname{Ly} \alpha$ ), the CTN factor can be computed readily as the ratio of the synthetic flux in the offline filter to that in Ly $\alpha$ filter (see Eq. (1)).

While calculation of the CTN factor from a spectrum may be computationally simple, knowing exactly which spectrum to use (i.e. which age and internal reddening) is far more complex. The region of interest is a bright young region of intense star formation where the age of young clusters ranges from 1 to $\sim 40 \mathrm{Myr}$ (Östlin et al. 2003). Studies of the central starburst 

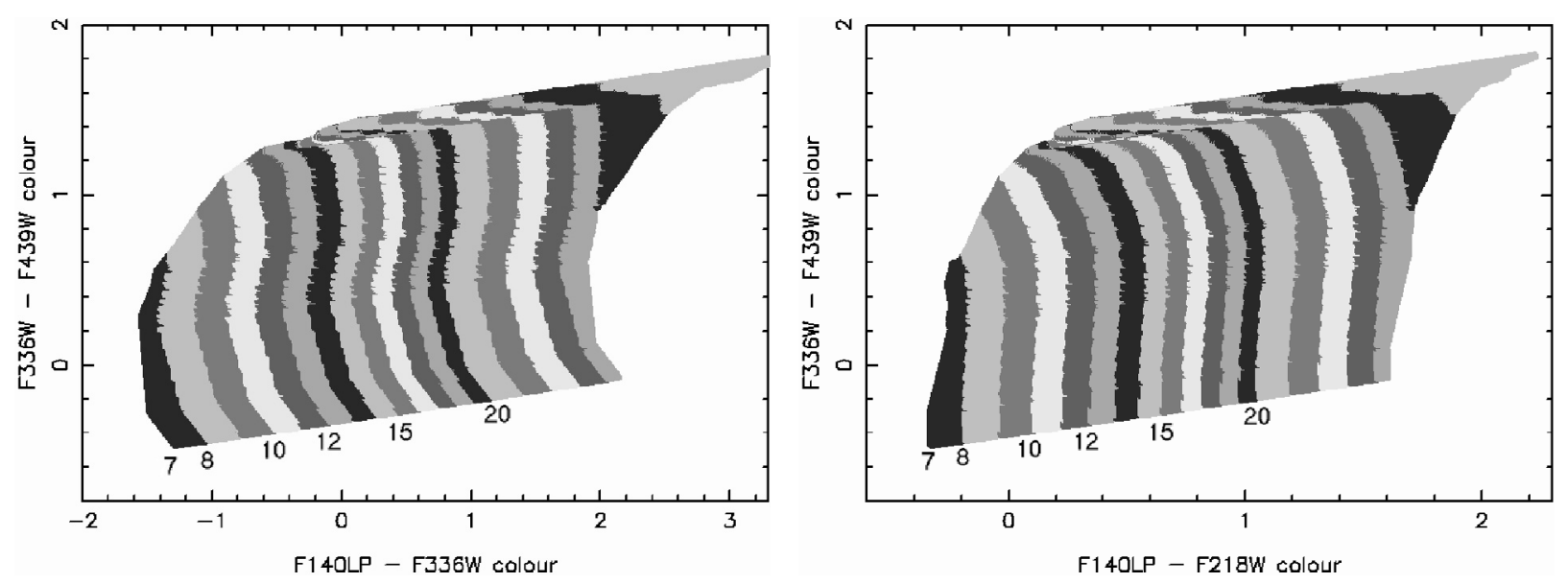

Fig. 5. Plots using two synthetic colours from the Starburst 99 models. The CTN factor is represented by colour in bins of width 1 unit. $\mathrm{CTN}=7-8$ is shown by the bottom left stripe and corresponds to the bluest UV/optical colours. CTN $=8-9$ in seen in the neighbouring band and so on. Left: F140LP-V218W vs. F336W-F439W. Right: F140LP-F336W vs. F336W-F439W. Colours are in Vega magnitudes.

region using the Balmer emission line decrement (e.g. Bergvall 1985; Raimann et al. 2000) and the UV continuum (Calzetti et al. 1994; Meurer et al. 1995; Buat et al. 2002) show the internal reddening to be very low: $E(B-V)<0.05$. Furthermore, groundbased longslit spectroscopy measuring $\mathrm{H} \alpha / \mathrm{H} \beta$ (Östlin et al. 2003) determined $E(B-V) \leq 0.20$ everywhere along the slit (aligned in the east-west direction).

Due to the well-known degeneracy in the effects of age and reddening, it is not possible to derive a non-degenerate relationship between a single colour (e.g. $U-B$ or $\beta$ ) and the CTN factor. Instead we began to look for a possible relationship between CTN factor and pairs of colours. Our goal was to establish a non-degenerate relationship between CTN factor and any pair of colours obtained from the SED, irrespective of the age of the starburst and the internal dust reddening. The procedure we implemented was as follows:

1. Begin with the full age range ( 1 to $900 \mathrm{Myr}$ ) of Starburst 99 spectra with Galactic reddening and HI absorption profiles applied.

2. Redden each spectrum using the SMC law (Prévot et al. 1984). Values of internal colour excess were in the range $E(B-V)=0$ to 0.4 in steps of $0.003 \mathrm{mag}$; extending beyond the maximum observed reddening of $0.2 \mathrm{mag}$.

3. Convolve each spectrum (many ages, many internal reddenings) with all instrument throughput profiles and integrate to obtain synthetic fluxes. From these, compute CTN factors and colours (convert colours to the Vega magnitude system).

4. Linearly interpolate between the age points to obtain extra colours and CTNs.

This procedure resulted in a large table of values: a CTN factor and all UV/optical colours for every step in $E(B-V)$ for every age. To look for relationships between the colours and CTN factors, we produced a series of colour-colour plots. For each possible age and reddening, a point was plotted in colourcolour space where the CTN factor was represented by the colour of the point. One plot was produced for every possible combination of HST colours. Each plot was studied to look for a combination of colours that corresponded to a single, nondegenerate value of the CTN factor. Two such diagrams are shown in Fig. 5.

It can be seen in Fig. 5 that for these combinations of colours, if the ordinate and abscissa are known, they can be used to find a single, non-degenerate value of the CTN factor. The F140LP-F336W vs. F336W-F439W combination was then chosen to produce a CTN factor map. While producing nice-looking plots, any set-ups involving the WFPC2/F218W filter were not chosen due to poor $\mathrm{S} / \mathrm{N}$ in this image. In areas of low $\mathrm{S} / \mathrm{N}$, the chosen images were smoothed using a $10 \times 10$ pixel box average filter. For each pixel in the aligned images, F140LP-F336W and F336W-F439W colours were calculated. These colours were converted to a CTN factor and written back to the corresponding pixel of a new image. This CTN factor map was then used to create the desired Ly $\alpha$ line-only image.

\section{Results}

The first CTN-factor map and the continuum subtracted Ly $\alpha$ image can be seen in Fig. 6. The green regions surrounding the starburst show the CTN factor to fall in the range 10.0 to 10.5 . This is consistent with the flat continuum slope which can also be seen in Fig. 3 where $\beta$ is shown to fall in the range -0.5 to 0.0 . In the centre the CTN factor falls to the minimum value permitted by the models: 7.2 (1 Myr, unreddened burst).

The first continuum subtracted Ly $\alpha$ image, in the right panel of Fig. 6 shows excellent qualitative agreement with features in the spectrum. Most notably:

- Ly $\alpha$ is seen in both emission and absorption along the slit. See also Figs. 2 and 4.

- Evidence for both emission and absorption can be seen in the regions immediately surrounding knot A. Bright emission and a small absorption hole appear in the continuum subtracted image. This is consistent with the P-Cygni profile, centred around $1228 \AA$ visible in Fig. 4.

- Emission is seen from the regions around knots C and D (consistent with the STIS spectrum). The lack of emission 

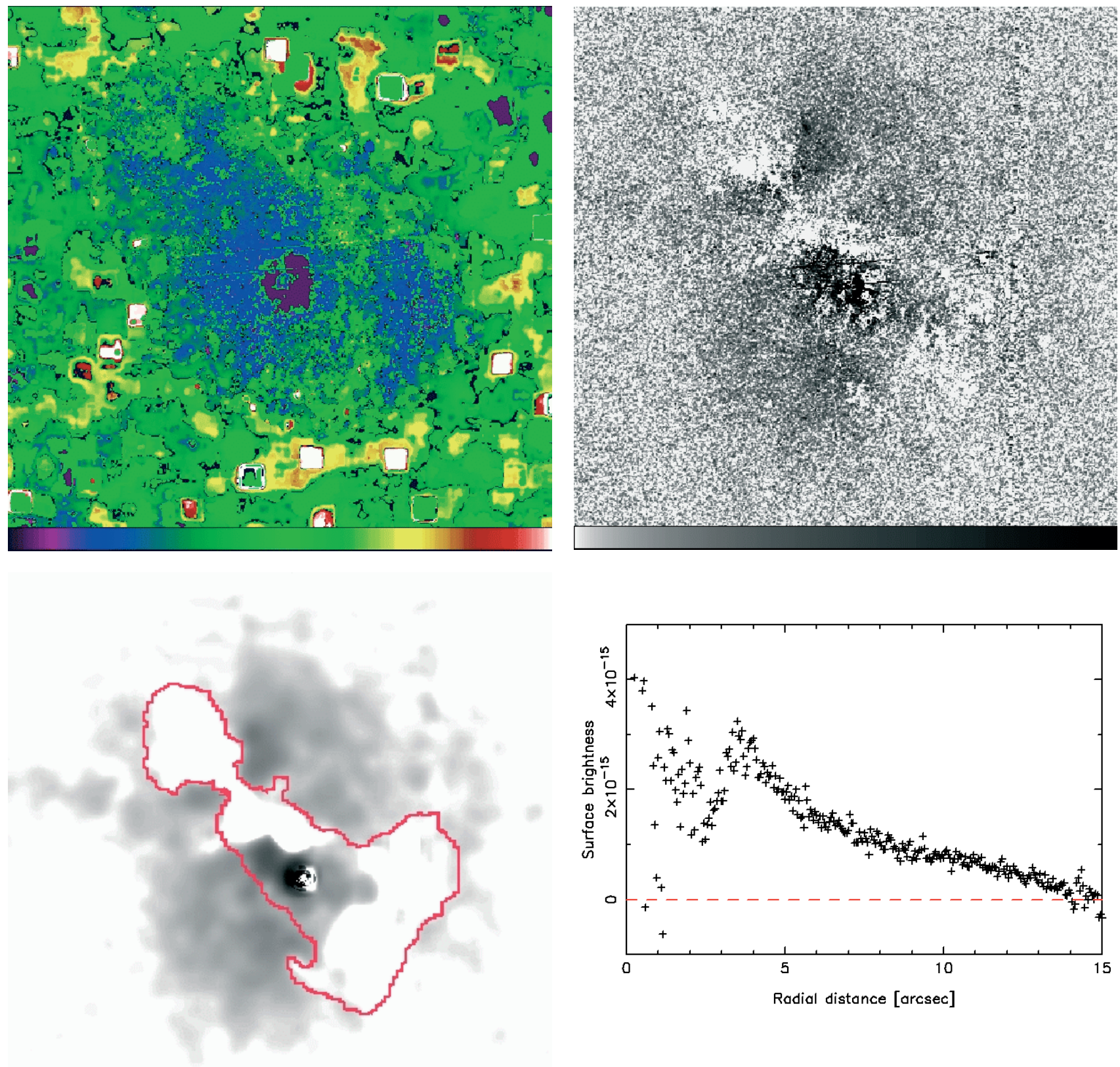

Fig. 6. Top left: map of CTN factor across the starburst region of ESO338-IG04. The intensity scale ranges from 7 in blue/purple to 14 in $\mathrm{red} /$ white. The midpoint green colour surrounding the starburst therefore corresponds to $\mathrm{CTN} \simeq 10.5$. A flat $(\beta=0)$ power-law continuum corresponds to $\mathrm{CTN}=10.33$. This image has been smoothed in the outskirts, where $\mathrm{S} / \mathrm{N}$ is low using a $10 \times 10$ pixel boxcar filter. Top right: Ly $\alpha$ image using the CTN map to the left for the continuum subtraction. The inverted log scale means emission is black and absorption white. Ly $\alpha$ escapes from the centre due to outflowing gas and is observed in diffuse regions where photons escape after HI scatterings. Bottom left: map of the Ly $\alpha$ absorption and emission, smoothed using an adaptive filtering algorithm. The red line indicates the border of the mask used to examine the central and diffuse regions individually. The smoothed version clearly shows knots A and C as spatially separate Ly $\alpha$ sources. The size of the images is $15 \times 15^{\prime \prime}$. Bottom right: radial distribution of Ly $\alpha$ flux in ESO 338-IG04 in units of erg s${ }^{-1} \mathrm{~cm}^{-2} \mathrm{arcsec}^{-2}$. Points in the innermost $0.5^{\prime \prime}$ fall above the plot. The profile shows a peak in Ly $\alpha$ surface brightness at $3.5^{\prime \prime}$ from the centre, corresponding to the diffuse emission regions seen in the top right and bottom left panels.

from these regions was one of the serious inadequacies of the $\beta$-map technique.

- Absorption is seen around knots B and E. Furthermore, the CTN factor map shows a decrease in CTN factor in the region of these knots, which is consistent with the continuum flux decreasing towards $\operatorname{Ly} \alpha$ in these knots as can be seen in the spectral image (Figs. 2 and 4).

- Although this is not covered by the STIS spectrum, diffuse emission is detected from large regions outside the central starburst. 

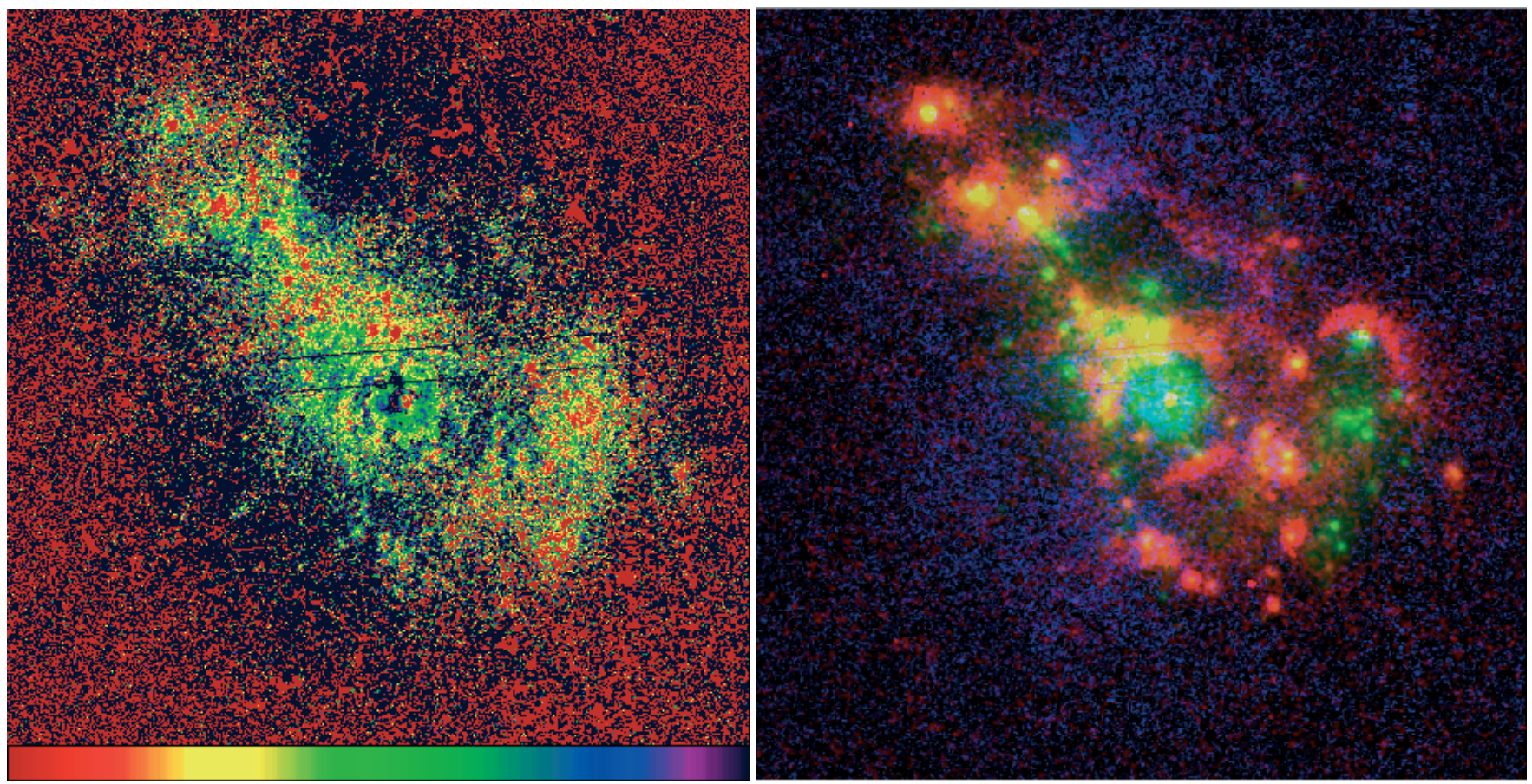

Fig. 7. Left: Ly $\alpha$ equivalent width map - negative scale - cuts levels are -50 to $+50 \AA$. Regions of high equivalent width show up in dark colours. Particularly visible are the diffuse emission regions outside the starburst region. Much local structure can be seen, particularly around knot A and the other bright continuum sources. Right: a false colour image: red shows the [OIII] STIS image; green shows the UV continuum F140LP image; blue the continuum subtracted Ly $\alpha$ image. The cuts levels are set to boost interesting features.

Quantitative photometry was performed on the continuum subtracted Ly $\alpha$ image and a total Ly $\alpha$ flux $f_{\text {Ly } \alpha, \text { TOT }}=194 \times$ $10^{-14} \mathrm{erg} \mathrm{s}^{-1} \mathrm{~cm}^{-2}$ was obtained. This is the sum of all the flux within a radius of $15^{\prime \prime}$, at which distance the Ly $\alpha$ surface brightness falls to zero. Ly $\alpha$ images were then masked with a $10 \times 20$ " elliptical mask, centred on the central star cluster (knot A). This corresponds to the size and shape of the aperture of the IUE satellite, from which the integrated Ly $\alpha$ flux and equivalent width of ESO 338-IG04 was presented in Giavalisco et al. (1996). These authors claim a Ly $\alpha$ flux of $123 \times 10^{-14} \mathrm{erg} \mathrm{s}^{-1} \mathrm{~cm}^{-2}$. Analysis of our continuum subtracted Ly $\alpha$ image reveals a Ly $\alpha$ flux of $f_{\mathrm{Ly} \alpha, \mathrm{IUE}}=134 \times$ $10^{-14} \mathrm{erg} \mathrm{s}^{-1} \mathrm{~cm}^{-2}$, perfectly consistent, considering the accuracy of the IUE. At a distance of $37 \mathrm{Mpc}$, this corresponds to Ly $\alpha$ luminosity $L_{\mathrm{Ly} \alpha}=3.17 \times 10^{41} \mathrm{erg} \mathrm{s}^{-1}$. The surface brightness profile of the Ly $\alpha$ emission line can be seen in Fig. 6. This profile was created by integrating the flux outwards from the central star cluster in concentric circles.

Using the CTN map, the F140LP image was renormalised to the continuum level at $\operatorname{Ly} \alpha$, and used together with the online image to create an equivalent width map. This map can be seen in the left panel of Fig. 7. Using the same IUE aperture mask as before, the equivalent width of $\operatorname{Ly} \alpha$ in this aperture was calculated as $22.6 \AA$. This again corresponds very closely to the value of $26.2 \AA$ presented in Giavalisco et al. (1996). The equivalent width exceeds $200 \AA$ in the diffuse emission regions where there is little continuum.

The equivalent widths were investigated in detail in some of the absorption regions. Absorption equivalent widths in some regions around the brightest star clusters are as low as
$-65 \AA$, indicating that the HI absorption in these regions is damped.

The RGB composite shown in Fig. 7 is composed of the continuum subtracted Ly $\alpha$ image created with the assumed parameters, the F140LP ACS image continuum image, and the [OIII] STIS image. Colour channels are: Red - [OIII]; Green $\mathrm{UV}$; Blue - Ly $\alpha$. This is discussed in Sect. 6.

\section{Model dependencies and other considerations}

The results presented above utilised our assumed set of model spectra, based upon various parameters that relate to the properties of the target galaxy. The Starburst99 spectra we used were created for very low metallicity and Salpeter IMF, with an additional assumption of the SMC law being made to redden the spectra. Some parameters were chosen based upon various known facts about the target galaxy (e.g. the low metallicity and the IMF) and others were assumed based upon studies of similar galaxies (e.g. the SMC law for internal reddening). If we want to know to what extent our results can be trusted, it is important to know how the selection of individual model parameters may influence our studies.

Some parameters are easily tested by changing the set of model spectra. We used other Starburst99 synthetic spectra to investigate the effects of metallicity and IMF simply by plugging a different set of models into our code. Similarly, changing the assumed law for internal reddening law was simple to implement by making minor modifications to the software. We adopted the assumed set of model parameters $(Z=0.001$, Salpeter IMF, SMC law) as the "standard" set, to which we compared all our subsequent modifications. By changing one 
Table 1. Model parameters for consideration.

\begin{tabular}{cccc}
\hline \hline Red. law & Metallicity & IMF $\alpha$ & IMF $M_{\text {up }}$ \\
\hline SMC & 0.001 & -2.35 & 100 \\
\hline LMC & 0.001 & -2.35 & 100 \\
Cardelli & 0.001 & -2.35 & 100 \\
Calzetti & 0.001 & -2.35 & 100 \\
\hline SMC & 0.004 & -2.35 & 100 \\
SMC & 0.008 & -2.35 & 100 \\
SMC & 0.020 & -2.35 & 100 \\
SMC & 0.040 & -2.35 & 100 \\
\hline SMC & 0.001 & -3.3 & 100 \\
SMC & 0.001 & -1.5 & 100 \\
SMC & 0.001 & -2.35 & 30 \\
\hline
\end{tabular}

Notes: $\alpha: \mathrm{d} N \propto M^{-\alpha} \mathrm{d} M, M_{\text {low }}=1 M_{\odot}$.

parameter at a time, we recomputed CTN maps and used them to create continuum subtractions. We compared the individual pixel values of the modified CTN maps to those of the standard CTN map and performed the same quantitative photometry on the Ly $\alpha$ line-only images as described in Sect. 4. Masks isolating the central and diffuse regions were also created so that these components could be examined individually if necessary. The parameter space explored in this manner is listed in Table 1.

Other effects that were investigated include those of nebular emission lines, a different spatial distribution of the nebular emission with respect to the ionising continuum, continuous star formation, and the stellar Ly $\alpha$ absorption feature.

It is important to note that, while changing model parameters may have a quantitative effect on the $\operatorname{Ly} \alpha$ flux and equivalent width, no noticeable differences could be seen by eye in the continuum subtracted Ly $\alpha$ images. Morphologically, the images resemble one another.

\subsection{Metallicity and IMF parameters}

Table 1 shows the metallicities and IMFs we investigated. Examples of how these parameters affect individual pixels in the CTN map can be seen in the top two panels of Fig. 8. The value of each pixel in the "standard" map is represented by the abscissa of each point while the value of the pixel in the new CNT map, normalised by its standard value, is represented by the ordinate.

It can be seen from the top plot that increasing the metallicity from $Z=0.001$ to $Z=0.008$ (well above the measured value) introduces a slight downward trend in the CTN values. This effect was present for all metallicities shown in Table 1. Increasing the metallicity to extreme values of $Z=0.040$ produced similar plots but with the scatter in the ordinate increased. Selection of a metallicity of $Z=0.008$ resulted in a decrease in the Ly $\alpha$ flux to $f_{\mathrm{Ly} \alpha, \mathrm{IUE}}=113 \times 10^{-14} \mathrm{erg} \mathrm{s}^{-1} \mathrm{~cm}^{-2}$, a drop of $16 \%$.

The centre plot in Fig. 8 shows the effect of steepening the index of the power-law governing the IMF to $\alpha=-3.3$. The effect is very similar to that obtained by increasing the
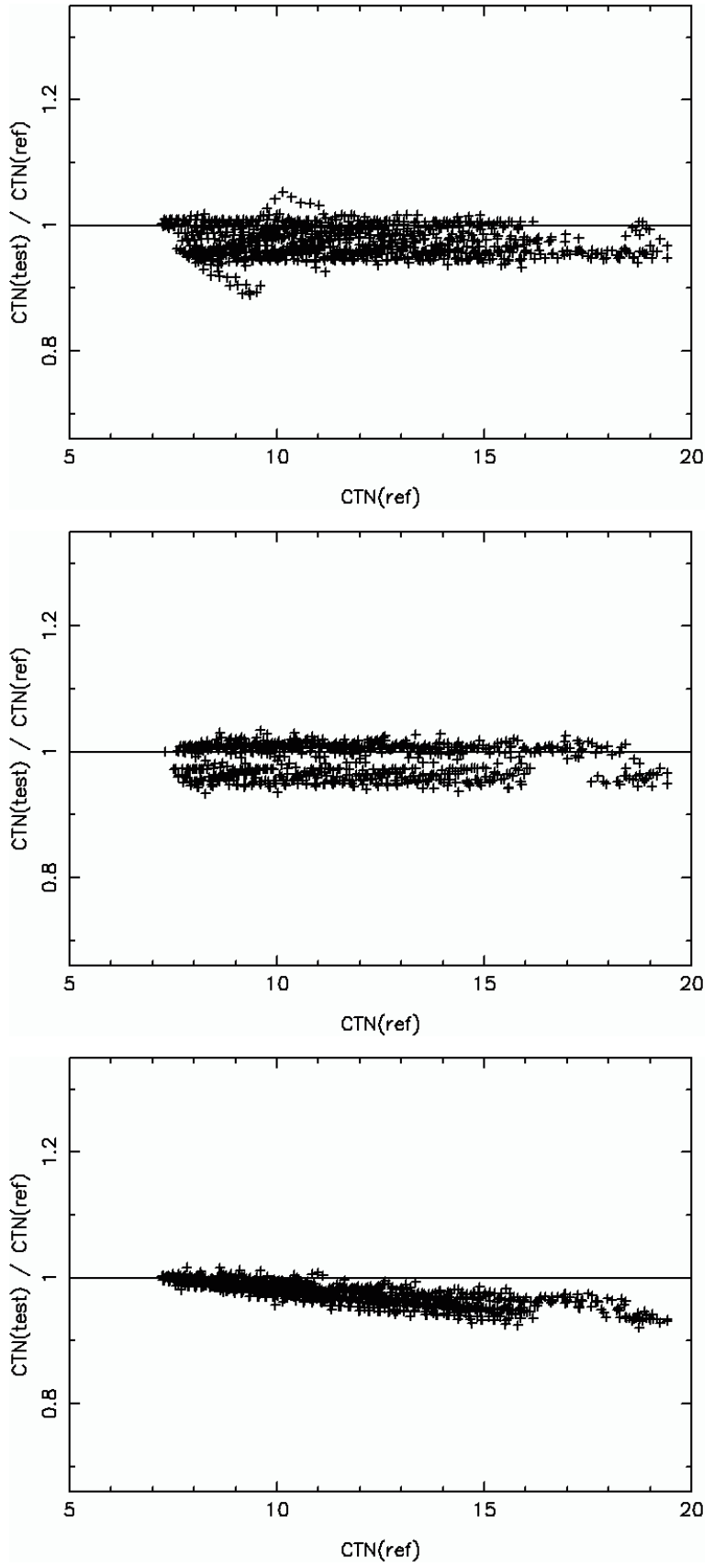

Fig. 8. Figures showing the effects of varying certain model parameters. Each point represents one pixel in the CTN map. The abscissa represents the value computed with the assumed set of model parameters. The ordinate shows the value of that pixel, normalised by the abscissa. I.e. the ordinate is unity if the pixel is unchanged by changing model parameters. The perturbed parameters are: top: metallicity $(Z=0.008)$; centre: IMF $(\alpha=-3.3)$; bottom: internal reddening law (LMC).

metallicity: a slight, systematic decrease of CTN. The same result was obtained when using the model set with $\alpha=$ $-2.35, M_{\text {up }}=30 M_{\odot}$. Both modifications to the IMF have similar effects on the CTN due to the fact that such modifications have similar effects on the massive stellar population: the relative fraction of massive stars is reduced, flattening the continuum in the UV. The use of a flatter IMF with $\alpha=-1.5$ had the 
opposite effect: the Ly $\alpha$ flux increased over that obtained with our standard parameter set to $168 \times 10^{-14} \mathrm{erg} \mathrm{s}^{-1} \mathrm{~cm}^{-2}$.

It can be seen that increasing metallicity and decreasing the relative number of hot young stars in the IMF have similar effects on the individual pixels in the CTN maps: a general, systematic decrease. This is an interesting result because one could naively assert that a process that flattens the continuum should lead to an increase in the CTN since the F140LP/F122M ratio will increase. However, as well as the CTN itself, metallicity, and IMF also affect the optical/UV colours, particularly the F140LP-F336W colour which represents the slope of the UV continuum. A redder SED leads to an increased CTN but also to a higher value for the F140LP-F336W colour, which serves to shift the points in the right panel of Fig. 5 to the right. Hence the colour obtained from the optical images may actually correspond to a lower CTN when the spectrum becomes flatter. It all depends upon whether the effect under consideration has a stronger impact on the CTN factor or on the optical colours.

\subsection{Extinction law}

In the method described in Sect. 3, we assumed the SMC law of Prévot et al. (1984) for the internal reddening law because in BCGs such as this larger graphite molecules are destroyed by the ionising radiation, producing an extinction law without any bump at $2200 \AA$, similar to that of the SMC (Mas-Hesse \& Kunth 1999). By the same method as for metallicity and IMF, we investigated how the choice of extinction law affects our study. The effect on the pixels in the CTN map of switching to the LMC law (Fitzpatric 1985) can be seen in the bottom panel of Fig. 8. The Galactic law of (Cardelli et al. 1989) and the Calzetti law (Calzetti 1997) showed a similar downward trend in CTN but to a greater extent.

This plot shows that, if the LMC law were applied instead of that of the SMC, the general trend would be to decrease the CTN factor at each pixel. The effect is more pronounced for larger CTN factors. Unlike in the cases of metallicity and IMF, this is what we would naively expect. The effect seen is attributed to the fact that the LMC law provides less extinction than the SMC at short wavelengths. This causes the spectra to which the LMC law was applied to have steeper UV continua than the same initial spectra reddened with the SMC law; the result is a lower CTN factor. This effect is also present in F140LP-F336W colour where the points are shifted towards the blue. The choice of extinction law has little impact on extinction at large wavelengths but varies by $\sim 50 \%$ at Ly $\alpha$ and therefore, the CTN is much more sensitive to the choice of extinction law than the optical colours are. In the Ly $\alpha$ line-only image, the flux in the IUE aperture was $121 \times 10^{-14} \mathrm{erg} \mathrm{s}^{-1} \mathrm{~cm}^{-2}$ when using the LMC law. This decrease in Ly $\alpha$ flux was also present when using the Cardelli law $\left(112 \times 10^{-14} \mathrm{erg} \mathrm{s}^{-1} \mathrm{~cm}^{-2}\right)$ and the Calzetti law $\left(76 \times 10^{-14} \mathrm{erg} \mathrm{s}^{-1} \mathrm{~cm}^{-2}\right)$.

\subsection{The stellar Ly $\alpha$ feature}

There has been a long controversy over the importance of $\operatorname{Ly} \alpha$ absorption in stellar atmospheres pertaining to observations of Ly $\alpha$ from nebulae; e.g. Valls-Gabaud (1993), Charlot \& Fall (1993). The synthetic starburst spectra comprise both stellar and nebular components with Ly $\alpha$ emission from stars (as from galaxies) subject to significant radiative transfer effects in their atmospheres. Atmospheres themselves are based upon empirical data that may include ISM absorption from the Milky Way in the spectra. Moreover there is a lack of data describing atmospheres of very low metallicity stars, and there is not a single $\mathrm{O}$ star for which Ly $\alpha$ can be observed due to the large ISM column. Depending on the wind properties, the Ly $\alpha$ feature can be in absorption, a filled-in P-Cygni profile, or in emission. For the hottest stars, we expect net equivalent widths of only around a few $\AA$ (Klein \& Castor 1978). While the current status of stellar atmosphere models may be sound, atmospheres are still not developed enough to allow us to determine the contribution of the stellar $\operatorname{Ly} \alpha$ feature to overall $\operatorname{Ly} \alpha$ from galaxies.

Using the best currently available model spectra, we attempted to investigate the effects of this feature. We adopted the latest modification of the SED@ synthetic spectra (M. Cerviño, private communication) based upon stellar evolutionary synthesis models. The stellar input spectra for these models were built with the TLUSTY and SYNSPEC codes of Lanz \& Hubeny (2003). These models predict Ly $\alpha$ assuming a planeparallel geometry, non-LTE, and full line-blanketing. The assumption of a plane-parallel geometry is often not appropriate for $\operatorname{Ly} \alpha$, which can form above the photosphere in the expanding outer envelope. We expect the models to produce Ly $\alpha$ absorption that is too strong, as the wind emission is neglected. Therefore the predicted stellar Ly $\alpha$ absorption equivalent width represents an upper limit to the observations. The spectra included stellar and nebular continuum emission for instantaneous burst with Salpeter IMF and solar metallicity. The age range available was 1 to $10 \mathrm{Myr}$. Without applying any internal reddening, Galactic reddening, or Galactic absorption to the spectra, we computed the CTN factor for these spectra. The stellar Ly $\alpha$ feature was then removed by interpolating linearly in between 1211 and $1221 \AA$ and the CTN factor recomputed. We found that, at ages less than $10 \mathrm{Myr}$, the removal of stellar Ly $\alpha$ feature decreases the CTN by no more than $1.8 \%$.

\subsection{Stellar, nebular, and emission line components}

In our analysis (Sect. 6) of the Ly $\alpha$ line-only image, diffuse emission is seen from regions surrounding the starbursting knots. The continuum flux in these regions is low and the stellar population is clearly aged in comparison to the bright continuum sources. The $\mathrm{H} \alpha$ equivalent width map (Bergvall \& Östlin 2002) shows equivalent widths in these regions of 300 to $400 \AA$. This stellar population is incapable of providing such a high $W(\mathrm{H} \alpha)$, and the ionising photons most likely come from the massive young star clusters that inhabit the central regions.

The Starburst99 dataset offers a choice of stellar-only or stellar + nebular spectra, which we used to subtract out the nebular-only component. We then used the independent components to investigate the effects of varying their relative contributions. Such a study is of importance since the spectra are 
devised for zero-dimensional objects, whereas in our images, the regions of strong nebular and ionising continua are clearly well resolved. Boosting the nebular component has the effect of lowering the CTN because in the 1200-1500 $\AA$ region (the region of the SED from which the CTN is computed) the nebular continuum is always flatter than the stellar. We found that to decrease the total Ly $\alpha$ flux by $10 \%$, we needed to boost the nebular continuum component by a factor of 20 . Such a boost in the nebular contribution would have a dramatic effect on $W(\mathrm{H} \alpha)$ but the effect on the CTN remains small. When measuring the age in the diffuse Ly $\alpha$ regions from our models (zero dimensional) for an instantaneous burst, we determine ages between 15 and $40 \mathrm{Myr}$. Such ages correspond to $W(\mathrm{H} \alpha)$ in the range 17.5 to $0.6 \AA$ in the same model set. Boosting the nebular component by a factor of 20 increases the measured $W(\mathrm{H} \alpha)=$ $17.5 \AA$ to $350 \AA$ - consistent with the $W(\mathrm{H} \alpha)$ map. For ages found in these regions, the nebular continuum in the region where the CTN is computed is around three orders of magnitude smaller than the stellar component; hence boosting the nebular component by reasonably large factors makes little difference to the CTN. Varying the relative contribution of the components has no effect on the Ly $\alpha$ map that is distinguishable by eye and has a negligible impact on the overall Ly $\alpha$ flux.

The Starburst99 dataset also offers the option of continuous star-formation as opposed to the instantaneous burst that we had assumed initially. While we believe the instantaneous burst to be a good approximation in the region of the current starburst, this may not necessarily be so for the underlying population which may contribute more strongly to the continuum in the regions from which we see diffuse Ly $\alpha$ emission. The continuous star-formation model also maintains a high $W(\mathrm{H} \alpha)$ for a much longer period, $(W(\mathrm{H} \alpha)>300 \AA$ at ages $>400 \mathrm{Myr}$ ). Using this model, we measured a Ly $\alpha$ flux decrease in the aperture corresponding to that of the IUE from 134 to $120 \times 10^{-14} \mathrm{erg} \mathrm{s}^{-1} \mathrm{~cm}^{-2}$. While it may fit the data rather well, we do not believe the continuous star-formation model on the grounds that clusters in these regions are no older than 40 Myr (Östlin et al. 2003).

Where the starburst is young, nebular emission lines may strongly affect the luminosity of a galaxy in certain wavebands; hence, the optical colours may be determined by the presence of nebular emission lines, not simply the continuum as previously assumed. The broad WFPC2 filters cover a large fraction of the optical domain and, from a galaxy such as ES0 338-IG04, this we can expect strong emission lines which may fall within our filters (e.g. the $F 555 W$ filter transmits the strong $\mathrm{O}[\mathrm{III}]$ lines). Since the model spectra we used do not include nebular line emission, we investigated how our results may, or may not, be influenced by the addition of lines to the spectra.

The Mappings III photoionisation code of Kewley et al. (2004, in prep.) was used to model nebular emission lines for a number of different burst ages via the Starburst99 interface. CTN factors and optical colours for all our filter possibilities were computed with and without the inclusion of nebular emission lines. The Ly $\alpha$ line was not included since the definition of CTN requires its absence.
For a burst of $5 \mathrm{Myr}$, the nebular emission lines did not affect the CTN by more than $1 \%$ or either of our selected colours by more than $0.05 \mathrm{mag}$. Nebular line emission was therefore not considered important for the study of this galaxy with the data that we have available.

\subsection{Possible departure from a single stellar population}

We also examined how sensitive the results are to the assumption of a single stellar population. Our concern originated from the possibility that the underlying stellar population may contaminate the optical colours of the pure starburst. We assumed that the old population may contribute $10 \%$ of the flux in the B-band and used the 900 Myr Starburst 99 spectrum as a template for this old population. For each age, we computed a new spectrum comprising the standard spectrum for that age, plus that of the old population normalised to $10 \%$ of the flux of the standard spectrum at $4000 \AA$. Again this modification produced a Ly $\alpha$ line-only image that was visually indistinguishable from that produced with the standard model parameters. In the IUE aperture, this modification produced a Ly $\alpha$ flux of $122 \times 10^{-14} \mathrm{erg} \mathrm{s}^{-1} \mathrm{~cm}^{-2}$; a decrease of $9 \%$.

Due to the very low UV flux of the old population, it has no discernible effect on the CTN and acts only to redden the computed optical colours. The effect was most noticeable for young ages in the $F 336 \mathrm{~W}-\mathrm{F} 439 \mathrm{~W}$ colour where it amounted to an increase of $\sim 0.1 \mathrm{mag}$ for a $1 \mathrm{Myr}$ unreddened burst. The effect of the underlying population was considered not to be an issue in the analysis of ESO 338-IG04.

\section{Analysis and discussion}

The two-colour technique described in Sect. 3.1 proves an excellent method of subtracting the continuum from the line in our ACS imaging survey. Disentangling the degenerate effects of age and reddening relies upon the sampling of the continuum slope in critical regions. In our technique we use one colour that is very sensitive to the reddening (F140LP-F336W, sampling the UV continuum slope $\beta$ ) and one colour that is sensitive to age ( $F 336 W-F 439 W$, sampling the $4000 \AA$ discontinuity). Not only does the qualitative comparison of the continuum subtracted image compare very well with the STIS spectra along the slit, but quantitative photometric results compare well with previous values of the Ly $\alpha$ flux and equivalent width.

In addition, an important point is that our results are not highly model dependent. Qualitatively, the line-only Ly $\alpha$ images were indistinguishable from one another, each showing the same features of absorption and emission and each showing the diffuse emission regions. Moreover, when comparing fluxes, all were in agreement to better than $50 \%$, even when parameter space well outside of the known constraints was investigated (eg, IMF $\alpha=-3.3$ or twice solar metallicity).

ESO 338-IG04 is a well-studied galaxy so these data can be compared with a wide array of other studies, against which our results can be tested. 


\subsection{The Ly $\alpha$ emission}

The false-colour map, (Fig. 7) shows how Ly $\alpha$, UV continuum, and [OIII] compare spatially. [OIII] emission can be used to gain insight into hardness of the ionising flux since it maps twice-ionised oxygen.

There are several noteworthy features in this image. The hot young star clusters, very bright in the UV continuum, appear as the bright green blobs. These are the regions where the continuum flux is high and the slope is steep due to the young stellar population, which is the reason why so many of the star clusters appear in green. All of the regions surrounding the green and yellow star clusters show [OIII] emission to some degree. Here the UV flux from the cluster is still strong enough to twice ionise oxygen at large distances from the UV signature of the cluster, an effect seen very strongly around knots B and F.

Knot $\mathrm{A}$ is another interesting case. Nearly the whole region around knot A shows up in either cyan or green, indicating not only a strong UV continuum but also a positive flux of Ly $\alpha$. This is consistent with the STIS spectrum of this knot. Ly $\alpha$ escape from this region due to the cluster driving an outflow of the local neutral ISM, giving rise to the P Cygni profile that can be seen around $1228 \AA$ in Fig. 4. In the spectrum, Ly $\alpha$ can be seen in absorption and emission, bluewards and redwards of $1228 \AA$, respectively. Just to the west of knot A, the cyan (Ly $\alpha$ and UV) becomes yellow (UV and O[III]), as the Ly $\alpha$ flux falls off but oxygen is still twice ionised.

Diffuse emission regions are very evident surrounding the starburst regions. In these regions the continuum flux is very low and Ly $\alpha$ equivalent widths exceed $200 \AA$. We explain this as $\operatorname{Ly} \alpha$ photons escaping the HI region either by diffusing to a region where the optical depth to infinity is less than unity or by scattering into the wing of the emissivity profile. These diffuse emission regions are very important for the overall line luminosity. As can be seen from the surface brightness profile, they dominate the luminosity output since the scattering mechanism is responsible for the majority of the $\operatorname{Ly} \alpha$ escape.

Analysis of the strong absorbing regions shows the Ly $\alpha$ equivalent width to be around $-65 \AA$, indicating damped absorption due to a static covering of neutral hydrogen. This value for the equivalent width in absorption compares well with estimates of equivalent widths of Ly $\alpha$ absorption in other BCGs. Cursory inspection of the GHRS spectra of SBS 0335-052 presented in Thuan \& Izotov (1997) and of I Zw 18 presented in Kunth et al. (1994) show Ly $\alpha$ equivalent widths of $\lesssim-50 \AA$ and $\lesssim-45 \AA$, respectively.

\subsection{Ly $\alpha$ correlations with other properties}

Östlin et al. (2003) mapped the age of the star clusters in this galaxy, the youngest having ages $\lesssim 2 \mathrm{Myr}$. These are the regions from which we expect Ly $\alpha$ to be trapped if the local ISM is not configured so as to allow its escape. Towards the west of the galaxy, this age map shows many massive, very young clusters which coincide exactly with the absorption from knots E, B, and the surrounding regions. Other young clusters are marked in Östlin et al. (2003) to the south and southeast of the central

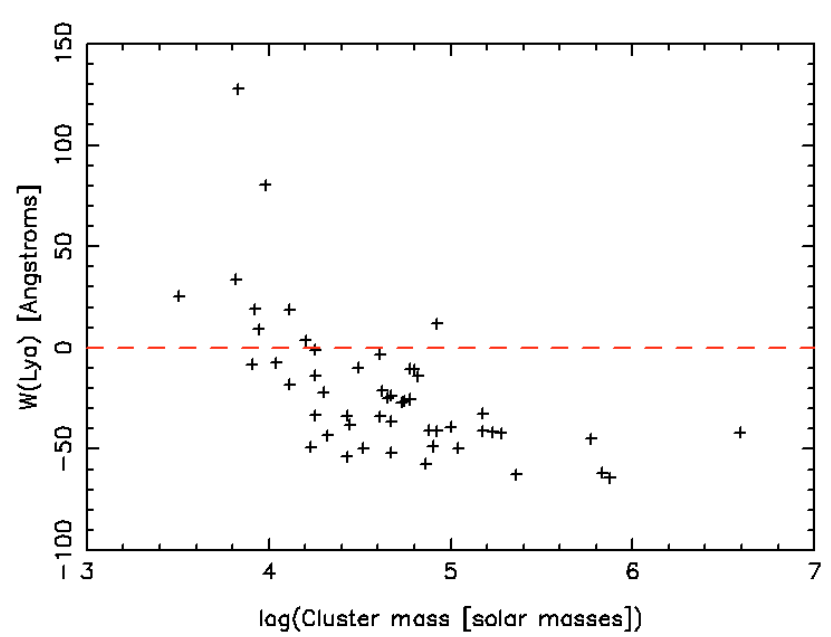

Fig. 9. Equivalent width of $\operatorname{Ly} \alpha$ in $\AA$ for a $3 \times 3$ pixel box around young clusters $(<30 \mathrm{Myr})$ vs. $\log$ cluster mass in $M_{\odot}$.

cluster. They also appear as absorption features in our continuum subtraction.

We compared the current results with those from Östlin et al. (2003) on the age distribution of compact star clusters. We selected the young ( $<30 \mathrm{Myr}$ ) sub-population and looked for systematic relationships between their properties (age, photometric mass, luminosity, colour, radial distance from the centre) and the local Ly $\alpha$ emission or absorption fluxes and equivalent widths. We note that we are comparing regions which are coincident on the $2 \mathrm{D}$ projected image and that are not necessarily spatially coincident in 3D.

Tenorio-Tagle et al. (1999) and Mas-Hesse et al. (2003) suggested that Ly $\alpha$ emission from starburst is expected to be a function of evolutionary and geometrical effects. In this model, pure or P-Cygni Ly $\alpha$ emission can be expected only if we are looking into the ionised cone. No Ly $\alpha$ should be expected along lateral sightlines that view the surrounding HI. We find no correlation between the Ly $\alpha$ escape/absorption or equivalent width and the age of the comparison cluster; hence we currently find no support for the evolutionary model. We stress, however, that Ly $\alpha$ escape is convolution of many parameters, not simply cluster age, and that geometry may provide the scatter that masks the trend.

The only clear trend is seen when comparing equivalent width and luminosity or mass: the more luminous (or massive) the cluster, the smaller the $E W$ is on average (and in fact it is predominantly negative, see Fig. 9). This serves to reinforce the discovery that Ly $\alpha$ escape is not regulated by the output UV luminosity from the cluster. This trend is expected if one assumes that the more massive clusters are associated with more gas, thus a larger neutral hydrogen column density.

\section{3. $L y \alpha$ and other kinematical tracers}

The ionised gas kinematics of this galaxy have been explored through Fabry-Perot interferometry, described in Östlin et al. (1999, 2001). This study revealed a complex and perturbed, almost chaotic velocity field. Photometric studies have shown the tail to the eastern side to contain a distinctly different 
stellar population from the rest. The authors explained this as the result of a merger that was the triggering mechanism for the current starburst. The merger may be responsible for triggering the starburst, producing the $\operatorname{Ly} \alpha$ photons, and is also responsible for the irregular ISM kinematics that allow these locally produced photons to leave the galaxy. One striking result of this study was the discovery that in the region of diffuse Ly $\alpha$ emission north of knot $\mathrm{A}$, the ionised gas is redshifted with respect to the stellar population. Regions south of knot A are similarly blueshifted. This could either point to a bipolar outflow of the ISM or the presence of a second kinematical component with a centre very close to knot $\mathrm{A}$ and kinematic axis in the northsouth direction. If real, this axis has the same orientation as the one along which we find for diffuse $\operatorname{Ly} \alpha$ emission.

A UVES spectrum of knot A discussed in Östlin et al. (2005 in prep.) shows that the ionised gas around this knot has at least two components: one which is redshifted by $\sim 25 \mathrm{~km} \mathrm{~s}^{-1}$ and one blueshifted by $\sim 40 \mathrm{~km} \mathrm{~s}^{-1}$. In addition, NaI D absorption lines which probably probe the neutral ISM, are blueshifted by $\sim 20 \mathrm{~km} \mathrm{~s}^{-1}$ compared with the stellar component. This suggests that gas is flowing towards us along the line of sight and is consistent with the P-Cygni interpretation of the Ly $\alpha$ emission around knot A. South of knot A the UVES spectrum reveals an ionised component blueshifted relative to knot A but also components redshifted by 100 to $200 \mathrm{~km} \mathrm{~s}^{-1}$, showing the complex nature of the medium.

The VLA HI mapping of ESO 338-IG04 and its companion also suggests a recent strong interaction between the two galaxies (Cannon et al. 2004). Although the HI map has a much lower resolution than the ACS images, it suggests that the regions with strong diffuse $\operatorname{Ly} \alpha$ emission north and south of knot A coincide with a region where the HI column density is smaller $\left(N(\mathrm{HI})<5 \times 10^{21} \mathrm{~cm}^{-2}\right)$ than elsewhere in the galaxy. The other strong diffuse $\operatorname{Ly} \alpha$ region to the west of knot A has $N(\mathrm{HI})>7 \times 10^{21} \mathrm{~cm}^{-2}$. In the region near knot A, although we stress again that the resolution makes a comparison with a point source even more uncertain, the column density is $N(\mathrm{HI}) \approx 6 \times 10^{21} \mathrm{~cm}^{-2}$.

All these column densities would be high enough to produce damped absorption of $\operatorname{Ly} \alpha$, something we do see in parts of the central starburst, despite its extremely low dust content. That any emission at all comes from the central UV-bright knots must be attributed to a clumpy ISM and to velocity shifts of neutral gas relative to the photon-producing regions. Note, however, that velocity shifts of $\mathrm{HI}$ (as well as $\mathrm{H} \alpha$ discussed above) over the galaxy are relatively small.

Synthesis of these kinematical tracers leaves an incomplete picture. The Fabry-Perot and UVES spectra hint that in the regions of diffuse $\operatorname{Ly} \alpha$ emission, the ISM may be subject to a rather slow large-scale outflow of gas (and hence allowing the escape of Ly $\alpha$ photons) but also reveal a highly perturbed medium. Conversely, the HI data show the neutral ISM to be subject only to very small velocity shifts over the whole galaxy (although the resolution is much poorer than the scales explored here), indicating the diffuse $\operatorname{Ly} \alpha$ emission may be the result of a clumpy medium, perturbed on a much smaller scale.
Table 2. Ly $\alpha$ Fluxes and equivalent widths from the various components.

\begin{tabular}{lll}
\hline \hline Region & $\begin{array}{l}\text { Sum of flux } \\
\left(10^{-14} \mathrm{erg} \mathrm{s}^{-1} \mathrm{~cm}^{-2}\right)\end{array}$ & $\begin{array}{l}E W \\
(\AA)\end{array}$ \\
\hline Centre positive & 93 & 47 \\
Centre negative & -163 & -78 \\
\hline Centre total & -70 & -17 \\
Diffuse & 204 & 113 \\
\hline Total & 134 & 22.6 \\
\hline
\end{tabular}

Notes: values in this table refer only to fluxes in the IUE aperture.

\subsection{The diffuse emission}

It has been shown in Sect. 5 that our results are not highly dependent upon the model parameters chosen. One striking result of the photometric study was that over $70 \%$ of the total Ly $\alpha$ originates from the diffuse emission regions.

Our unreddened Ly $\alpha$ flux in the IUE aperture amounts to $134 \times 10^{-14} \mathrm{erg} \mathrm{s}^{-1} \mathrm{~cm}^{-2}$. However, this comprises a combination of positive and negative contributions and the net contribution from the central burst region is negative. We can divide the total Ly $\alpha$ flux into 3 spatial components: central regions with positive Ly $\alpha$ flux, central regions with negative Ly $\alpha$ flux, and the region outside, i.e. from where we see diffuse emission. Table 2 shows the fluxes and equivalent widths in these regions.

Had it not been for the positive diffuse emission, this galaxy would not have any Ly $\alpha$ detected by the IUE satellite.

We compare these numbers with the $\mathrm{H} \alpha$ flux obtained from ground-based narrowband imaging (Bergvall \& Östlin 2002). Of the total $\mathrm{H} \alpha$ flux $\left(f_{\mathrm{H} \alpha}=290 \times 10^{-14} \mathrm{erg} \mathrm{s}^{-1} \mathrm{~cm}^{-2}\right.$, Östlin et al. 2001; Gil de Paz et al. 2003), 87\% comes from the region covered by our Ly $\alpha$ observations. Of the $\mathrm{H} \alpha$ flux in this region approximately $35 \%$ comes from the diffuse region and the rest from the central regions. The uncertainty on these quantities is about $\pm 5 \%$ and arises due to the very different spatial resolution of the HST/Ly $\alpha$ and the ground-based $\mathrm{H} \alpha$ images. Of course we do not have the resolution needed to differentiate the $\mathrm{H} \alpha$ flux in the regions showing positive and negative Ly $\alpha$ flux.

Correcting for Galactic extinction, $\operatorname{Ly} \alpha / \mathrm{H} \alpha=3.3$ for the diffuse region. The Ly $\alpha$ photons in this region could either be locally produced by ionising flux from the central burst (as there are no UV-bright sources in the diffuse region, see Östlin et al. 2003) or be due to Ly $\alpha$ photons diffusing out from the centre after multiple scatterings. The former alternative appears more realistic since we see $\mathrm{H} \alpha$, which is not subject to multiple scattering, in the diffuse region; and moreover, the HI column density in the diffuse region is high with little velocity difference with respect to the central burst (Cannon et al. 2004). If we assume that the internal reddening in the diffuse region is similar to that in the centre $(E(B-V)=0.05$, see also Östlin et al. 2003) and that the SMC reddening law is most realistic, this would lead to a corrected $\mathrm{Ly} \alpha / \mathrm{H} \alpha$ ratio of 6.6 , or about half the value predicted from case A recombination. To reconcile these numbers, either we have to assume that the internal reddening in the diffuse region is a factor of $>2$ higher than in the centre, or that each $\operatorname{Ly} \alpha$ photon has suffered, on 
average, $\gtrsim 2$ additional scatterings, whereby the total Ly $\alpha$ intensity has been reduced by the 2-photon process. The latter appears very reasonable given the large neutral hydrogen column density. Hence, the diffuse emission is best explained by ultraviolet photons escaping the central burst through a porous medium and ionising hydrogen in the diffuse regions, after which Ly $\alpha$ photons are scattered a few times before escaping.

In the central region, absorption dominates emission. The predicted equivalent width of $\operatorname{Ly} \alpha$, disregarding any absorption processes, is on the order of $200 \AA$, whereas damped absorption may lead to negative values $<-50 \AA$. Thus to create a net absorption more than $80 \%$ of the photons with wavelength near $\lambda_{\mathrm{em}}=1216 \AA$ have to be absorbed by static (or nearly static) neutral hydrogen. That we see any pixels with positive Ly $\alpha$ must be attributed to the porosity of the ISM and local velocity differences between the ionised gas and neutral medium in front of it along the line of sight. Looking at the central $\mathrm{H} \alpha$ flux and attenuating it by Galactic reddening and an additional internal reddening of $E(B-V)=0.05$ using the SMC law, we would expect to see a flux of approximately $1000 \times 10^{-14} \mathrm{erg} \mathrm{s}^{-1} \mathrm{~cm}^{-2}$ if $\mathrm{HI}$ absorption was neglected. The central $\mathrm{Ly} \alpha$ photons that do reach us amount to $93 \times 10^{-14} \mathrm{erg} \mathrm{s}^{-1} \mathrm{~cm}^{-2}$, or about $9 \%$. This is consistent with more than $80 \%$ of the photons being destroyed. The pixel scale of ACS/SBC corresponds to a linear-projected scale of $5 \mathrm{pc}$ at the distance of $37 \mathrm{Mpc}$. Possibly the number of positive central Ly $\alpha$ photons would be higher with even better spatial resolution.

Taking the $\mathrm{H} \alpha$ flux from the area corresponding to the ACS images, we corrected this flux for Milky Way reddening and for internal reddening using the SMC law with $E(B-V)=0.05$. Assuming case A recombination, we computed the total Ly $\alpha$ flux produced to be $3860 \times 10^{-14} \mathrm{erg} \mathrm{s}^{-1} \mathrm{~cm}^{-2}$. By comparing this value to the detected Ly $\alpha$ flux (corrected for Galactic extinction) of $194 \times 10^{-14} \mathrm{erg} \mathrm{s}^{-1} \mathrm{~cm}^{-2}$ we obtain an escape fraction of $5 \%$. This escape fraction is an order of magnitude lower than what one would derive $(44 \%)$ by assuming internal reddening only.

\subsection{Implications for high-z surveys}

Our technique of performing a continuum subtraction is dependent upon careful modeling of the SED. We notice that the region of the SED imaged by our offline filter (central wavelength: $1400 \AA$; rectangular width: $250 \AA$ ) corresponds almost exactly to the region of the SED of a galaxy at redshift of around 4, as imaged by a broadband Johnson $R$ filter. Malhotra \& Rhoads (2002) used this broadband filter to subtract continuum from their sample of galaxies in the redshift range 4.37 to 4.57 using a variety of online narrowband filters. Hence, their continuum filter maps a very similar part of the restframe SED to ours. The Ly $\alpha$ galaxies in their sample were found to have higher than expected equivalent widths with a median value of $400 \AA$. It is therefore interesting to point out that, when we assumed a flat continuum, we derived a Ly $\alpha$ flux for ESO 338-IG04 of $430 \mathrm{erg} \mathrm{s}^{-1} \mathrm{~cm}^{-2}$ and equivalent width of $72 \AA$ - over 3 times higher than the values obtained through SED modeling. Making naive assumptions about the continuum slope caused us to vastly overestimate the Ly $\alpha$ equivalent width. To perform accurate high- $z$ surveys, we suggest it may be necessary to at least employ more offline filters so as to accurately model the continuum.

\section{Conclusions}

We have presented high resolution $\operatorname{Ly} \alpha$ imaging of the local starburst galaxy ESO 338-IG04 based on observations with the HST and the solar blind channel of ACS, complemented by available WFPC2 data. We show that a careful continuum subtraction is crucial and demonstrate that we have a technique in place to subtract the continuum, provided the necessary supporting data are available. In our analysis of Starburst 99 synthetic spectra, we were able to disentangle the effects of age and internal reddening by carefully modeling the evolution of the UV continuum slope and $4000 \AA$ discontinuity. We present a total Ly $\alpha$ line flux of $f_{\text {Ly } \alpha \text {, TOT }}=194 \times 10^{-14} \mathrm{erg} \mathrm{s}^{-1} \mathrm{~cm}^{-2}$ and demonstrate the photometric validity of our technique by comparison with STIS spectroscopy and the IUE data presented in Giavalisco et al. (1996). Our Ly $\alpha$ flux of $134 \times$ $10^{-14} \mathrm{erg} \mathrm{s}^{-1} \mathrm{~cm}^{-2}$ matches that obtained with the IUE satellite of $123 \times 10^{-14} \mathrm{erg} \mathrm{s}^{-1} \mathrm{~cm}^{-2}$. Within the errors associated with the IUE, these values are in perfect agreement. We also find a total $\operatorname{Ly} \alpha$ equivalent width of $22.6 \AA$, again in agreement with the value obtained with the IUE. We find extensive evidence for resonant photon scattering in diffuse Ly $\alpha$ emission regions that surround the central starburst. These regions dominate the total output luminosity of the line and equivalent widths in here exceed $200 \AA$. If it had not been for the diffuse emission, this galaxy would not have been seen as a net emitter of Ly $\alpha$ e.g. in IUE spectra. By comparing the $\mathrm{Ly} \alpha$ and $\mathrm{H} \alpha$ fluxes in these regions, we calculated that each detected Ly $\alpha$ photon had suffered 2 additional scatterings, reducing the net Ly $\alpha$ emission via two-photon emission. We estimate that of all the Ly $\alpha$ photons produced, only 5\% leave the galaxy. We find that the Ly $\alpha$ line traces the kinematics and perturbed morphology of ESO 338-IG04, correlating closely with other tracers of kinematics. In all, this galaxy provides an excellent laboratory for studying the physics of $\operatorname{Ly} \alpha$ emission, displaying a variety of mechanisms: emission through P-Cygni features from the central knot (A), damped absorption, leakage of central Ly $\alpha$ photons through paths with small optical depth, and diffuse emission. While our technique relies entirely upon the use of synthetic spectra, it is not highly dependent upon the choice of parameters (metallicity, IMF, etc.) nor on the choice of internal reddening law. We discuss the implications of careful SED modelling for the interpretation of photometric Ly $\alpha$ data, with reference to Ly $\alpha$ emitters at high- $z$. We find that if the continuum slope is not carefully modelled then fluxes and equivalent widths may be overestimated by factors of $\sim 3$.

Acknowledgements. M.H. and G.Ö. acknowledge the support of the Swedish National Space Board (SNSB) and the Swedish Research Council (V.R.). J.M.M.H. was supported by Spanish MEC under grants AYA2001-3939-C03-02 and AYA2004-08260-C03-03. We thank M. Cerviño for his help with the high resolution continuum models and J. M. Cannon \& E. D. Skillman for their contribution to this study. This work was supported by HST grant GO-9470 
from the Space Telescope Science Institute, which is operated by the Association of Universities for Research in Astronomy, Inc., under NASA contract NAS5-26555.

\section{References}

Bergvall, N. 1985, A\&A, 146, 269

Bergvall, N., \& Östlin, G. 2002, A\&A, 390, 891

Buat, V., Burgarella, D., Deharveng, J. M., \& Kunth, D. 2002, A\&A, 393, 33B

Charlot, S., \& Fall, S. M. 1993, ApJ, 415, 580

Cannon, D., Skillman, E., Kunth, D., et al. 2004, ApJ, 608, 768

Calzetti, D., Kinney, A. L., \& Storchi-Bergmann, T. 1994, ApJ, 429, 582

Calzetti, D. 1997, AJ, 113, 162

Cardelli, J. A., Clayton, G. C., \& Mathis, J. S. 1989, ApJ, 345, 245

Dickey \& Lockman, 1990, ARA\&A, 28, 215

Fitzpatrick, E. L. 1985, ApJ, 299, 219

Frye, B., Broadhurst, T., \& Benítez, N. 2002, ApJ, 568, 558

Fujita, S. S., Ajiki, M., Shioya, Y., et al. 2003, AJ, 125, 13

Fynbo, J. U., Möller, P., \& Thomsen, B. 2001, A\&A, 374, 443

Giavalisco, M., Koratkar, A., \& Calzetti, D. 1996, ApJ, 466, 831

Gil de Paz, A., Madore, B. F., \& Pevunova, O. 2003, ApJS, 147, 29

Haiman, Z., \& Spaans, M. 1999, ApJ, 518, 138

Hu, E. M., Cowie, L. L., \& McMahon, R. G. 1998, ApJ, 502, L99

Kewley, L. J., Dopita, M. A., Evans, I., et al. 2004, in prep.

Klein, R. I., \& Castor, J. I. 1978, ApJ, 220, 902

Kunth, D., Lequeux, L., Sargent, W. L. W., \& Vaillefond, F. 1994, A\&A, 282, 709

Kunth, D., Mas-Hesse, J. M., Terlevich, E., et al. 1998, A\&A, 334, 11

Kunth, D., Leitherer, C., Mas-Hesse, J. M., Östlin, G., \& Petrosian, A. 2003, ApJ, 597, 263
Lanz, T., \& Hubeny, I. 2003, ApJS, 146, 417

Leitherer, C., Schaerer, D., Doldader, J. D., et al. 1999, ApJS, 123, 3

Mar, D. P., \& Bailey, G. 1995, PASA, 12, 239

Malhotra, S., \& Rhoads, J. E. 2002, ApJ, 565, 71

Masegosa, J., Moles, M., \& Campos-Aguilar, A. 1994, ApJ, 420, 576

Mas-Hesse, J. M., \& Kunth, D. 1999, A\&A, 349, 765

Mas-Hesse, J. M., Kunth, D., Tenorio-Tagle, G., et al. 2003, ApJ, 598, 858

Meurer, G. R., Heckman, T. M., Leitherer, C., et al. 1995, AJ, 110, 2665

Neufeld, D. A. 1991, ApJ, 370, L85

Östlin, G., Bergvall, N., \& Rönnback, J. 1998, A\&A, 335, 85 (ÖBR98)

Östlin, G., Amram, P., Masegosa, J., Bergvall, N., \& Boulesteix, J. 1999, A\&AS, 137, 419

Östlin, G., Amram, P., Bergvall, N., et al. 2001, A\&A, 374, 800

Östlin, G., Zackrisson, E., Bergvall, N., \& Rönnback, J. 2003, A\&A, 408,887

Ouchi, M., Shimasaku, Z., Furusawa, H., et al. 2003, ApJ, 582, 60

Partridge, R. B., \& Peebles, P. J. E. 1967, ApJ, 147, 868

Pavlovsky, C., Biretta, J., Boffi, F., et al. 2002, ACS Instrument Handbook, Version 3.0 (Baltimore: STScI)

Prévot, M. L., Lequeux, J., Prévot, L., Maurice, E., \& Rocca-Volmerange, B. 1984, A\&A, 132, 389

Pritchet, C. J. 1994, PASP, 106, 1052

Raimann, D., Storchi-Bergmann, T., Bica, E., Melnick, J., \& Schmitt, H. 2000, MNRAS, 316, 559

Salpeter, E. E. 1955, ApJ, 121, 161

Schlegel, D. J., Finkbeiner, D. P., \& Davis M. 1998, ApJ, 500, 525

Tenorio-Tagle, G., Silich, S. A., Kunth, D., Terlevich, E., \& Terlevich, R. 1999, MNRAS, 309, 332

Thuan, T. X., \& Izotov, Y. I. 1997, ApJ, 489, 623

Valls-Gabaud, D. 1993, ApJ, 419, 7 